\title{
Cancer Molecular Analysis Project
}

National Cancer Institute

\section{Source}

National Cancer Institute. Cancer Molecular Analysis Project. NCI Thesaurus. Code C48274.

Through the Cancer Molecular Analysis Project (CMAP), NCl facilitates the identification and evaluation of molecular targets by integrating comprehensive molecular characterizations of cancer and making the data and infrastructure publicly accessible. CMAP permits investigators to 1) Discover molecular targets, 2) Assess their validity and interaction with other targets, 3) Determine if there are therapeutic agents that can act on specific targets, 4) Screen for possible toxicity, and 5) Determine whether there are clinical trials evaluating these agents. 